\title{
Preliminary Observation and Significance of Changes on Rash of Skin Prick Test during SLIT
}

\author{
Weinian Lin*, Jinchao Lin, Jun Liao, Runfeng Chen, Zhiwei Huang, Xiaodong Zhang, Wen Lin \\ Department of Otorhinolaryngology, Quanzhou First Affiliated Hospital of Fujian Medical University, Quanzhou, China \\ Email: *linwn99@163.com
}

How to cite this paper: Lin, W.N., Lin, J.C., Liao, J., Chen, R.F., Huang, Z.W., Zhang, X.D. and Lin, W. (2018) Preliminary Observation and Significance of Changes on Rash of Skin Prick Test during SLIT. International Journal of Otolaryngology and Head \& Neck Surgery, 7, 209-213.

https://doi.org/10.4236/ijohns.2018.74022

Received: June 26, 2018

Accepted: July 27, 2018

Published: July 30, 2018

Copyright $\odot 2018$ by authors and Scientific Research Publishing Inc. This work is licensed under the Creative Commons Attribution International License (CC BY 4.0).

http://creativecommons.org/licenses/by/4.0/

\begin{abstract}
Objective: To observe the changes on skin wheal and erythema of skin prick test for the patients with allergic rhinitis during SLIT. Methods: Since March 2010 the 103 cases of SLIT attacked by allergic rhinitis patients, divided into four age groups, respectively measured the diameter of skin wheal and erythema before treatment, six months, one year and 2 years after SLIT. The data were analyzed by analysis of variance method; $\mathrm{P}<0.01$ showed the difference was statistically significant. Results: The results showed that the most changes of skin erythema diameter were statistically significant in N1, N2, N3 age group during test observation compare with the data before SLIT ( $\mathrm{p}<$ 0.01 ); and the most changes of allergen wheal diameter were not statistically significant, but the N4 group had no significant change of wheal and erythema diameter. Conclusion: Although most of the skin test wheal did not change significantly during the treatment of SLIT, the erythema reaction decreased to a certain extent, indicating that the intensity of histamine release may be reduced during the treatment.
\end{abstract}

\section{Keywords}

Rhinitis, Allergy, Immunotherapy, Skin Tests, Therapeutic Evaluation

\section{Introduction}

Skin prick test (SPT) and detection of serum specific IgE are the main methods for the diagnosis of allergic rhinitis, combined with medical history, clinical symptoms and nasal signs [1] [2] [3] [4]. Specific immunotherapy (SIT) is increasingly considered as a main first-line treatment for allergic rhinitis [1] [2] [3] [4]. Sublingual immunotherapy (SLIT) is widely used because of its convenience, operability and good patient compliance. In this study, we investigated the changes and significance of test results of wheal and erythema in allergen SPT in 
the treatment of SLIT.

\section{Methods}

\subsection{Clinical Data}

A total of 103 patients (72 males and 31 females), with complete data, underwent SLIT at our hospital from March 2010 to February 2016, and were recruited in this study. All case accorded with the guidelines for the diagnosis and treatment of allergic rhinitis (2015, Tianjin, China) were on the indication of with moderate to severe AR classification, and the duration of the disease was 1 - 16 years, with an average of lyears and 8 months, The subjects were divided into four groups, with 22 patients ( 17 males and 5 females) in N1 group ( $4-8$ years old), 38 patients (27 males and 11 females) in N2 group (8 years and 1 month to 14 years old), 35 patients ( 24 males and 11 females) in N3 group (14 years and 1 month to 40 years old), and eight patients (4 males and 4 females) in N4 group ( $>40$ years old).

\subsection{Test Method}

The standard allergen SPT was performed on the skin inside forearm of patients with suspected allergic rhinitis. Common inhaled allergen provided by ALK-Abello was used, comprised include common ragweed, mugwort, compound mite (house dust and dust), Penicillium notatum, Humulus scandens pollen, tree compound II (willow, poplar, elm), cockroach, cocoon silk, cat hair and dog hair allergen liquid and cross reaction control substance. The normal skin inside forearm was selected and wiped with cotton ball dipped in saline water, the standard allergen solution was dripped onto the skin at $2 \mathrm{~cm}$ intervals, then the skin was pricked by an independent lancet, the forearm was exposed for 20 minutes, and the test results were analyzed.

According to the guidelines for the diagnosis and treatment of allergic rhinitis (2015, Tianjin, China) [1], the skin index SI is used as one of the diagnostic indicators of allergic rhinitis, so the SI was adopted, and the maximum and minimum diameters (the vertical line at the midpoint of maximum diameter was taken) of wheal of mite allergen and histamine prick point were measured respectively, to calculate their average wheal diameters of mite allergen and histamine prick point (the maximum and minimum diameters addition are equal to 2 equal to the average diameter), and the ratio of the two was SI, which was classified into four grades: $0.3 \leq \mathrm{SI}<0.5:+, 0.5 \leq \mathrm{SI}<1.0:++, 1.0 \leq \mathrm{SI}<2.0:+++$ SI $\geq$ 2.0: ++++ .

\subsection{Test Data Acquisition}

In clinical practice, the patients with allergic rhintis were selected with the SI for skin test to compound mite allergen liquid was +++ to ++++ . After the patients with positive reactions to other allergens were excluded, the specific SLIT with Dermatophagoides farinae drops was conducted. The results of SPT 
to mite allergens before treatment, as well as at 6 months, 1 year and 2 years after treatment were recorded. The maximum diameters of wheal and erythema of the mite allergen prick point were also recorded along with the clinical symptoms.

\subsection{Patient Management}

All patients and their families were given medication notes at the first visit. Follow-up files were established, contact information was noted, regular follow-up interviews were conducted to guide and resolve patients' care needs, the results of allergen SPT were detected, medication for home care was prescribed, environmental health was advised, dose adjustment and adverse drug reaction were noted, all of which improved the treatment compliance. The patients were asked to discontinue oral or local antihistamine and hormone drugs for one week before next SPT examination.

\subsection{Statistical Analysis}

In this study, SPSS 13.0 statistical software was used for all analyses. The quantitative data were expressed as mean \pm standard deviation $(\bar{x} \pm s)$. The data were approximately normally distributed, and repeated measures analysis of variance was used. $\mathrm{p}<0.01$ was considered as significant difference.

\section{Results}

In this study, after eliminating antihistamines in the case of pricked erythema, as Table 1 shows that the diameter of erythema at almost every observation time in the N1, N2 and N3 groups was significantly changed $(\mathrm{p}<0.01)$ as compared to the data before treatment, except for the date in N2 group at 2 years after treatment and the date in N3 group at 1 years after treatment. However, most changes on the diameter of allergen wheal were not statistically significant, and the diameters of erythema in the N4 group did not significantly change during SLIT at each time point.

Table 1. Changes in the diameter of wheal blush of SPT in the four groups at different time points (unit: $\mathrm{cm}$ ).

\begin{tabular}{|c|c|c|c|c|c|c|c|c|}
\hline \multirow[b]{2}{*}{ Group } & \multicolumn{2}{|c|}{ First time } & \multicolumn{2}{|c|}{6 months } & \multicolumn{2}{|c|}{12 months } & \multicolumn{2}{|c|}{24 months } \\
\hline & Wheal & Erythema & $\begin{array}{l}\text { Wheal } \\
\text { (p value) }\end{array}$ & $\begin{array}{l}\text { Erythema } \\
\text { (p value) }\end{array}$ & $\begin{array}{l}\text { Wheal } \\
\text { (p value) }\end{array}$ & $\begin{array}{l}\text { Erythema } \\
\text { (p value) }\end{array}$ & $\begin{array}{l}\text { Wheal } \\
\text { (p value) }\end{array}$ & $\begin{array}{l}\text { Erythema } \\
\text { (p value) }\end{array}$ \\
\hline N1 & $0.84 \pm 0.24$ & $2.01 \pm 0.86$ & $\begin{array}{c}0.64 \pm 0.28 \\
(0.024)\end{array}$ & $\begin{array}{c}1.33 \pm 0.66 \\
(0.001)^{\star}\end{array}$ & $\begin{array}{c}0.72 \pm 0.22 \\
\quad(0.111)\end{array}$ & $\begin{array}{c}1.42 \pm 0.63 \\
(0.001)^{\star}\end{array}$ & $\begin{array}{c}0.77 \pm 0.13 \\
(0.83)\end{array}$ & $\begin{array}{c}1.56 \pm 0.48 \\
(0.008)^{\star}\end{array}$ \\
\hline N3 & $0.97 \pm 0.35$ & $2.42 \pm 0.83$ & $\begin{array}{c}0.84 \pm 0.30 \\
(0.008)^{\star}\end{array}$ & $\begin{array}{c}1.54 \pm 0.64 \\
(0.001)^{\star}\end{array}$ & $\begin{array}{c}0.88 \pm 0.33 \\
(0.019)\end{array}$ & $\begin{array}{c}2.00 \pm 0.70 \\
(0.026)\end{array}$ & $\begin{array}{c}0.90 \pm 0.34 \\
(0.203)\end{array}$ & $\begin{array}{c}1.51 \pm 0.57 \\
(0.001)^{\star}\end{array}$ \\
\hline $\mathrm{N} 4$ & $1.06 \pm 0.42$ & $2.46 \pm 1.37$ & $\begin{array}{c}0.98 \pm 0.36 \\
(0.072)\end{array}$ & $\begin{array}{c}1.55 \pm 0.64 \\
(0.173)\end{array}$ & $\begin{array}{c}1.00 \pm 0.44 \\
(0.634)\end{array}$ & $\begin{array}{c}1.95 \pm 1.34 \\
(0.415)\end{array}$ & $\begin{array}{c}0.88 \pm 0.41 \\
(0.024)\end{array}$ & $\begin{array}{c}1.75 \pm 0.90 \\
(0.031)\end{array}$ \\
\hline
\end{tabular}

Note: ${ }^{\star} \mathrm{p}<0.01$. 


\section{Discussion}

SIT is one of first-line treatments for AR [1] [2] [3] [4]. In 2013, the World Allergy Organization (WAO) stated in the sublingual specific immunotherapy position paper that SLIT can be used as a therapeutic strategy for initial and early respiratory diseases [3]. Dermatophagoides farinae Drops (Chang di), as the main product of SLIT for AR, has been used for more than ten years in China, and its biological stability and clinical efficacy are generally recognized. In this study, Dermatophagoides farinae Drops (Chang di) treatment of patients with AR sensitive to dust mite achieved an ideal effect by alleviating clinical symptoms, reducing the occurrence of AR complications, and effectively reducing the use of antihistamines and other drugs [4] [5] [6] [7].

There is no simple and acceptable indicator to determine the clinical efficacy of specific immunity in patients with AR. The possible tests that may help to evaluate the efficacy include sIgE, sIgG4, cytokines, antigen specific T-cell detection, nasal provocation test, allergen exposed compartment, histamine release test, etc. The allergen SPT only included histamine release test. In clinical practice, skin sensitivity test included the adjacent reactions of wheal and erythema. The positive result of skin sensitive test with erythema reaction is generally believed to be stronger than simple wheal reaction. We found that during SLIT with mite allergen, most patients showed reduction of clinical symptoms and nasal mucosa pale edema, which became ruddy. However, most of the results of prick test in clinical practice were not significantly improved. The erythema around wheal improved in some patients. However, this improvement was not found in all patients throughout the treatment period. Whether the intensity of histamine release improved in some patients during treatment remains unknown. Because SPT is affected by numerous conditions, more extensive clinical data for comparative analysis are needed.

In this study, after eliminating antihistamines in the case of pricked erythema, the data at almost every observation time point in the N1, N2 and N3 groups were significantly changed as compared to the data before treatment. However, most changes in allergen wheal were not statistically significant, and the diameters of erythema in the N4 group did not significantly change during SLIT at each time point, and this may be related to too little sample. With the progress of SLIT, clinical symptoms improved but the wheal reaction of allergen SPT did not significantly change, indicating that the results of erythema reaction of histamine release intensity significantly reduced. Moreover, and there was no significant change in the $\mathrm{N} 4$ group ( $>40$ years old).

A recent study showed that in a group of $4-60$ years old patients with AR, who underwent SLIT (Dermatophagoides pteronyssinus and Dermatophagoides farinae Drops) for six months, the clinical nasal symptoms were significantly improved, the onset was rapid, with an average onset time of 14 weeks, the levels of dust mite and house dust mite specific IgG4 significantly increased, but the levels of serum specific IgE did not significantly change as compared to the con- 
trol group. Specific IgG4, a tolerogenic antibody, competed with serum specific IgE for the same allergen binding site, which prevented the release of inflammatory mediators [2] [6] [7]. One study [8] have shown that these IgG antibodies not only have the function of "blocking antibodies" but also have the ability to prevent SIgE mediated allergen histamine release, which seems to provide a clue as to answer why the reduction in the erythema diameter of some age groups found in our study.

SIT is the process of inducing immune tolerance by giving increasing doses of allergenic allergen vaccine to patients with AR [2] [6]. When the patient is re-exposed to the corresponding allergen, the clinical symptoms are absent or reduced. However, the sensitization of allergens was unchanged, perhaps because immune tolerance and minor histamine release played clinical therapeutic effects. Clinical trials showed that the younger the age, the more pronounced were the erythema changes of histamine release intensity. These preliminary results need to be confirmed in a large sample size clinical study.

\section{Conflicts of Interest}

The authors declare no conflicts of interest regarding the publication of this paper.

\section{References}

[1] Group of Experts on Rhinology (2015) Chinese Guidelines for Diagnosis and Treatment of Allergic Rhinitis. Chinese Journal of Otorhinolaryngol Head and Neck Surgery, 51, 6-18.

[2] Cheng, L. (2016) Allergen-Specific Immunotherapy as the First-Line Treatment for Allergic Rhinitis. Journal of Otolaryngology and Ophthalmology of Shandong University, 30, 1-2.

[3] Cheng, L. (2017) Some Issues Concerning the Prevention and Treatment of Allergic Rhinitis. Journal of Clinical Otorhinolaryngology Head and Neck Surgery, 31, 1-2.

[4] Canonica, G.W., Cox, L., Pawankar, R., Baena-Cagnani, C.E., Blaiss, M., Bonini, S., et al. (2014) Sublingual Immunotherapy: World Allergy Organization Position Paper 2013 Update. World Allergy Organization Journal, 7, 6. https://doi.org/10.1186/1939-4551-7-6

[5] Wang, D.H., Chen, L., Cheng, L., Li, K.N., Yuan, H., Lu, J.H., et al. (2013) Fast Onset of Action of Sublingual Immunotherapy in House Dust Mite-Induced Allergic Rhinitis: A Multicenter, Randomized, Double-Blind, Placebo-Controlled Trial. Laryngoscope, 123, 1334-1340. https://doi.org/10.1002/lary.23935

[6] Canonica, G.W., Bousquet, J., Casale, T., et al. (2009) Sub-Lingual Immunotherapy: World Allergy Organization Position Paper 2009. Allergy, 64, 1-59. https://doi.org/10.1111/j.1398-9995.2009.02309.x

[7] Jutel, M., Agache, I., Bonini, S., Burks, A.W., Calderon, M., Canonica, W., et al. (2016) International Consensus on Allergen Immunotherapy II: Mechanisms, Standardization, and Pharmacoeconomics. The Journal of Allergy and Clinical Immunology, 137, 358-368. https://doi.org/10.1016/j.jaci.2015.12.1300

[8] Dearon, M. (1997) Negative Regulation Regulation of Mast Cell Activation by Receptors for IgG. International Archives of Allergy and Immunology, 113, 138-141. https://doi.org/10.1159/000237528 